\title{
Use of Semantic Interoperability to Improve the Urgent Continuity of Care in Danish ERs
}

\author{
Sine Nordstrøm Lambert ANDERSEN ${ }^{\mathrm{a}, 1}$, Cecilie Mørk BRANDSBORG ${ }^{\mathrm{a}}$, and \\ Louise PAPE-HAUGAARD ${ }^{\mathrm{a}}$ \\ ${ }^{a}$ Depart. of Health Science and Technology, Aalborg University, Denmark
}

\begin{abstract}
Reuse of patient data from prehospital electronic health record (EHR) to EHRs in emergency rooms is currently non-existing. In Danish EHRs, access to patient data recorded in prehospital settings is either not available or accessible in a PDF file. The amount of patient and administrative data registered at the prehospital unit is high and includes a rich representation of the accident, the patient and treatment. By applying emphasis framing to the representation of data, information overload can be diminished. Several international studies have investigated the suboptimal reuse of data within this field. Hence, the aim of this pre-study was to develop webservices based on emphasis framing to increase interoperability between the prehospital health record and the emergency room's EHR. In this study, requirements engineering and emphasis framing was applied. Iterative linear requirement specification process was chosen as a frame to address the aim. The five included phases were revisited due to the iterative nature of this study. Tools used in the requirement engineering process were semi structured interviews and direct observations. The pre-study resulted in 12 Fast Healthcare Interoperability Resources (FHIR) profiles using SNOMED CT terminology bindings. The profiles contained elements which represented primarily patient data recorded in the prehospital setting. The profiles were compared to a case representing the urgent continuity of care to validate their ability to standardize data from prehospital health records. Conclusively, FHIR profiles can be modelled to standardize prehospital urgent patient data to support the patient trajectory. With the applied emphasis framing, the clinical context and content have been maintained.
\end{abstract}

Keywords: Interoperability, prehospital care, Emergency room, FHIR, SNOMED CT

\section{Introduction}

At the site of incident or during transfer to the Emergency Room (ER), the prehospital unit (PU) is making several recordings to assess the patient, thus registrations in the electronic prehospital health record (PHR). When the patient is handed over to the ERpersonnel, few of the registrations have to be reassessed. The registrations are currently currently accessible in a PDF file or as handwritten notes. This PDF file is compiled when the paramedics are handing over the patient. Accessing the PDF file is a manual and time

\footnotetext{
${ }^{1}$ Sine N. L. Andersen, Depart. of Health Science and Technology, Aalborg University, Niels Jernes Vej 10, 9220 Aalborg Øst, Denmark; E-mail: sine.andersen95@gmail.com
} 
consuming task in an extreme critical workflow. Raeisi et al. [1] is also arguing that the handoff of the patient in the interface between PU and ER is crucial for further course.

Multiple studies showed that the communication between the PU and the ER was suboptimal [1-5]. A study focused exclusively on patient data to be communicated and shared across PU and ER [6]. Other studies developed IT-systems aiming at real time transmitting recorded patient data across PU and ER [7-10]. A solid digital infrastructure aiming at increasing data transfer across PU and ER could potentially improve the digital communication. [11,12]. One possible solution to support a digital infrastructure is simply a webservice platform. [13] Such an infrastructure can support the clinical requirement of only reusing time-relevant patient data [14], as reuse of data should not occur with outdated data.

The case below exemplified the continuity of care for the acute patient:

To investigate the objective of the study a patient case has been generated. The requirements to a realistic case was to illustrate the amount and richness of patient data recorded in PU. When meeting the requirements to the case, information overload might occur, demonstrating the relevance of emphasis framing. The case is validated through literature and a clinician.

** "A patient was cutting branches of a large tree in the garden, where the patient fell 2 meters down from the ladder. When the ambulance arrived, the patient was conscious but complained of pain in the right arm and at the right hip (VAS: eight out of ten). The patient had no known allergies but had well-treated type 2 diabetes. After the medical history was recorded, the ambulance rescuers triaged the patient. The patient's vital parameters were: Temp: $36.8^{\circ} \mathrm{C}$, HR: $111 / \mathrm{min}, \mathrm{RF}: 31 \mathrm{~min}, \mathrm{BP}: 150 / 100 \mathrm{mmHg}$, SpO2: $89 \%$ and GCS: 14. The patient had free airways but moderate shortness of breath. Due to the height of the fall, the patient was placed on a spine board with a collar to ensure stability. When the patient entered the ambulance, the ambulance rescuers treated the patient with morphine (10 mg, given intravenously). The patient was examined and the ambulance rescuers suspect a fracture of the hip and a fracture of the right forearm, which was entered as a note to the ER. The ambulance rescuers transported the patient to the ER." **

This pre-study investigates how emphasis framing can be applied when patient data have to be shared across two separate units, PU and ER, with heterogeneous IT-solutions.

\section{Methods and materials}

With the use of Requirements Engineering (RE), it was partly ensured that the requirements for the webservice were elaborated and specified, but most importantly, it was ensured that the webservice could ultimately be verified and validated on the basis of the requirements. In addition, RE ensured that the requirements met the needs identified by the stakeholders. [15] Stakeholders for this study were emergency nurses. Interviews and observations were conducted at Hjørring Hospital, Denmark and Aalborg University Hospital, Denmark.

Iterative linear requirements specification process (ILRSP) was chosen because the inputs created the basis for the requirements that had to be set, and it was therefore deemed essential to have these well-defined. [16] The phases of ILRSP was elicitation, analysis, documentation and validation of the requirements. [15] For the standardization, HL7 FHIR standard and SNOMED CT-terminology bindings were used. To standardize 
data, Forge (version 4.0.1) for HL7 FHIR (Firely Amsterdam, Bos en Lommerplein 280, 1055 RW Amsterdam, The Netherlands) was used.

\section{Results}

In the phase of requirements analysis, the information gathering and documentation process of the urgent patient care was investigated to identify which HL7 FHIR profiles were to be used. The identified profiles were investigated and a blueprint was created to form the architectural foundation of the project.

With the use of the developed 12 profiles, it was ensured that the data required by the stakeholders was standardized for further reuse. The 12 profiles were compared to the case from the Introduction to validate their ability to standardize data from PHR. The comparison can be seen in Table 1.

Table 1. The table compares the case from the introduction with the developed 12 profiles. the case is broken down into manageable bits which are linked to the profile in which the data is standardized.

\begin{tabular}{|c|c|}
\hline Case & FHIR profile \\
\hline A patient... & Patient \\
\hline $\begin{array}{l}\text {... is cutting branches of a large tree in the } \\
\text { garden, where the patient fell } 2 \text { meters down from } \\
\text { the ladder. }\end{array}$ & ClinicalImpressionVitalSignsTriage \\
\hline $\begin{array}{l}\text { When the ambulance arrives, the patient is } \\
\text { conscious but complains of pain in the right arm } \\
\text { and at the right hip (VAS: eight out of ten). }\end{array}$ & ClinicalImpressionVitalSignsTriage \\
\hline The patient has no known allergies... & ObservationAllergyIntolerance \\
\hline ... but has well-treated type 2 diabetes. & $\begin{array}{l}\text { ObservationCurrentMedicationUse } \\
\text { ObservationPriorIllness }\end{array}$ \\
\hline $\begin{array}{l}\text { The patient's vital parameters are: Temp: } 36.8^{\circ} \mathrm{C} \text {, } \\
\mathrm{HR}: 111 / \mathrm{min}, \mathrm{RF}: 31 \mathrm{~min}, \mathrm{BP}: 150 / 100 \mathrm{mmHg} \text {, } \\
\text { SpO2: } 89 \% \text { and } \mathrm{GCS}: 14 . \text { The patient has free } \\
\text { airways but moderate shortness of breath. }\end{array}$ & ClinicalImpressionVitalSignsTriage \\
\hline $\begin{array}{l}\text { Due to the height of the fall, the patient is placed } \\
\text { on a spine board with a collar to ensure stability. }\end{array}$ & ProcedureTreatment \\
\hline $\begin{array}{l}\text { When the patient enters the ambulance, the } \\
\text { ambulance rescuers treat the patient with } \\
\text { morphine (10 mg, given intravenously). }\end{array}$ & $\begin{array}{l}\text { MedicationAdministrationGivenMedicine } \\
\text { Medication } \\
\text { SubstanceActiveIngredient }\end{array}$ \\
\hline $\begin{array}{l}\text { The patient is examined and the ambulance } \\
\text { rescuers suspect a fracture of the hip and a } \\
\text { fracture of the right forearm. }\end{array}$ & ClinicalImpressionVitalSigns Triage \\
\hline
\end{tabular}

In the table, the information from the case was linked to the FHIR profile which contained the registration of that piece of information. All requested data from the stakeholders were illustrated in the case, and as the table showed, all the information in 
the case was contained in the developed profiles. Furthermore, the reuse of data from the PHR included the profiles Encounter, Organization and Practitioner.

\section{Discussion}

By utilizing the needs of the stakeholders, the study sought to prevent information overload. Therefore, further research of the information applicable for the personnel in the ER was necessary to counteract information overload. The findings of the study by Laker et al. (2018) [17] emphasize the importance of a compromise when deciding on the amount of data to reuse. Therefore, the use of emphasis framing has the potential to improve the quality of the treatment, though the decision making time may increase. To fully understand the benefits of using emphasis framing in this study, it will require implementation of the FHIR profiles in the webservice and user test of the EHR system when data is reused.

In the development of the 12 FHIR profiles, elements of the resources were excluded and elements that would be beneficial for prehospital data were not present. Currently, the FHIR base resources do not fully incorporate prehospital incidents and actions. The prehospital field is limited as the information that can be gathered is often predefined in the PHR. To standardize data from PRH more thoroughly and thereby obtain complete interoperability, more FHIR base resources specific to the prehospital field must be developed. The semantic interoperability would also be strengthened if SNOMED CT contained exact terminologies which reflect the information gathered in PHR. In this study, registrations from PHR are compelled to bind to two SNOMED CT terms and others are compelled to bind to an equivalent but not exact SNOMED CT term. Since the Danish PHR has predefined registrations, it would benefit the semantic interoperability if the registrations were defined in the SNOMED CT terminology database.

Further research within this context is necessary to evaluate the true effect of the developed FHIR profiles in a specific clinical setting. Further research could include an in-depth analysis of how more data from PHR could be reused in EHR as well as user tests of the EHR system when the FHIR profiles are implemented in the webservice.

To enhance semantic interoperability between PHR and EHR, 12 HL7 FHIR profiles containing SNOMED CT terminology bindings were developed. The profiles are developed using requirements engineering and emphasis framing which ensures the quality of the reused data. It is concluded that the FHIR profiles contain elements to standardize data from an urgent continuity of care based on the registrations in PRH. Thereby, the clinical content and context are maintained.

\section{References}

[1] Raeisi A, Rarani MA, Soltani F. Challenges of patient handover process in healthcare services: A systematic review. Journal of Education and Health Promotion. 2019 Sep;8(173):1-6.

[2] Cuk S, Wimmer H, Powell L. Investigating Problems Associated with Patient Care Reports and Transferring Data between Ambulance and Hospitals from the Perspective of Emergency Medical Technicians. Issues in Information Systems. 2017;18(4):16-26.

[3] Martin J, Ranney ML, Dorroh J, Asselin N, Sarkar IN. Health Information Exchange in Emergency Medical Services. Applied Clinical Informatics. 2018;9(4):884-891.

[4] Zhang Z, Sarcevic A, Bossen C. Constructing common information spaces across distributed emergency medical teams. Proceedings of the ACM Conference on Computer Supported Cooperative Work. 2017:934-947. 
[5] Meisel ZF, Shea JA, Peacock NJ, Dickinson ET, Paciotti B, Bhatia R, Buharin E, Cannuscio CC. Optimizing the patient handoff between emergency medical services and the emergency department. Annals of Emergency Medicine. 2015 Mar;65(3):310-7.

[6] Finnell JT, Overhage JM. Emergency medical services: the frontier in health information exchange. AMIA Annu Symp Proc. 2010;222-6.

[7] Nakae T, Kataoka H, Kuwata S, Iihara K. Smartphone-assisted prehospital medical information system for analyzing data on prehospital stroke care. Stroke. 2014 May;45(5):1501-4.

[8] Gaynor M, Myung D, Hashmi N, Shankaranarayanan G, Moulton S. An intelligent prehospital patient care system. International Journal of Electronic Healthcare. 2007 Feb;3(1):107-122.

[9] Gaynor M, Myung D, Gupta A, Moulton S. A standardised pre-hospital electronic patient care system. International journal of electronic healthcare. 2009;5(2):102-136.

[10] Anantharaman V, Swee Han L. Hospital and emergency ambulance link: using IT to enhance emergency pre-hospital care. International journal of medical informatics. 2001 May;61(2-3):147-161.

[11] Al-Harbi N, El-Masri S, Saddik B. An integration of Emergency Department Information and ambulance systems. Studies in Health Technology and Informatics. 2012;180:985-9.

[12] El-Masri S, Saddik B. Proposal of an end-to-end emergency medical system. Studies in health technology and informatics. 2011;169:349-353.

[13 ] Hirshon JM, Risko N, Calvello EJB, Stewart de Ramirez S, Narayan M, Theodosis C, O’Neill J. Health systems and services: the role of acute care. Bulletin of the World Health Organization. 2013 May;91(5):386-388.

[14] Gordon BD, Flottemesch TJ, Asplin BR. Accuracy Evaluation of Emergency Department Tracking System Timestamps. Ann. Emerg. Med. 2008 Nov;52(5):504-511.

[15] Arif SU, Khan Q, Gahyyur SAK. Requirements Engineering Process, Tools, Technologies and Methodologies. International Journal of Reviews in Computing. 2010;41-56.

[16] Mehmood M, Ijaz BB. A Review of Requirement Engineering Process Models. Journal of Architectural Engineering Technology. 2018 Aug;7(1):1-10.

[17] Laker LF, Froehle CM, Windeler, JB, Lindsell CJ. Quality and Efficiency of the Clinical DecisionMaking Process: Information Overload and Emphasis Framing. Production and Operations Management. 2018 Aug;27(12):2213-2225. 